\title{
The Density and Mass Distribution of Meteoritic Bodies in the Neighborhood of the Earth's Orbit ${ }^{1}$
}

\author{
Harrison Brown \\ California Institute of Technology \\ Pasadena, California
}

\begin{abstract}
A study of the frequency of meteorite falls in areas which have been highly populated throughout the last century indicates that the average rate of fall of meteorites on the earth probably lies between 0.32 and 1.0 falls $/$ year $10^{\circ} \mathrm{km}^{2}$. The frequency of fall as a function of type and mass has been studied, and it is shown that the distribution curves for stones and irons have similar shapes. The curves are also remarkably similar to those observed for asteroids. The observed relationships are used to calculate the impact frequency of meteoritic particles upon the earth and moon as a function of their size.
\end{abstract}

Introduction. There are many reasons for our wanting to know the density of meteoritic bodies in the neighborhood of the earth's orbit. Opik [1957] has recently computed the frequency of impact of bodies greater than 60 meters in diameter with the lunar surface. This study, which was undertaken before Professor Oopik's unpublished paper ['The Lunar Surface as an Impact Counter'] was brought to the present author's attention, gives estimates of impact frequencies with the moon and the earth of particles weighing 1 gram and more.2

The frequency of meteorite falls upon the earth. We can obtain an indication of the density of meteoritic bodies in the neighborhood of the earth's orbit by estimating the frequency of encounter of such bodies with the earth. At present approximately ten meteorites are seen to fall and are recovered each year over the earth. The number of observed falls has been

1 This paper was presented at the symposium conducted by the Committee on Space Research of the International Council of Scientific Unions at Nice, France, on January 11, 1960. This material, together with other papers presented at the Symposium, will appear in a volume to be published by the North-Holland. Publishing Co., Netherlands.

2 It has also been brought to the author's attention, since the delivery of this paper, that Gerald S. Hawkins has conducted a similar study, soon to be published in the Astronomical Journal. The computed mass distributions and frequencies of terrestrial fall are compatible with those found here. increasing rather steadily with time, in part as a result of the increasing numbers of human beings in the world and in part as a result of a growing interest in meteorites. It is important to ask: If all meteorites that actually reach the earth's surface were seen and recovered, how many would be counted each year?

We can estimate the frequency of meteorite impact on the earth's surface by studying the frequency of observed meteoritic falls in areas of the world which have had high rural population densities throughout the last century and in which there is a reasonably high probability that a recovered meteorite will eventually be reported. Three such areas are Japan, India, and Western Europe. Table 1 shows, in the lefthand columns, the number of meteorites that fell in these areas during the half-centuries 1840-1889 and 1890-1939, and in the right-hand columns, the density of fall in each area. For comparison, the corresponding figures are given for the United States. The errors shown are standard 'counting errors,' assuming random events.

It will be noted that the densities of fall in the three selected areas are in surprisingly close agreement. Although the total number of falls on a world-wide basis increased with time during this period, there is no obvious time-dependence in these particular areas. During the first half-century, 110 meteorites fell in these regions, whereas 100 were observed during the second. It is interesting that the observed density of fall 
HARRISON BROWN

TABLE 1. Meteorite Falls in Selected Areas

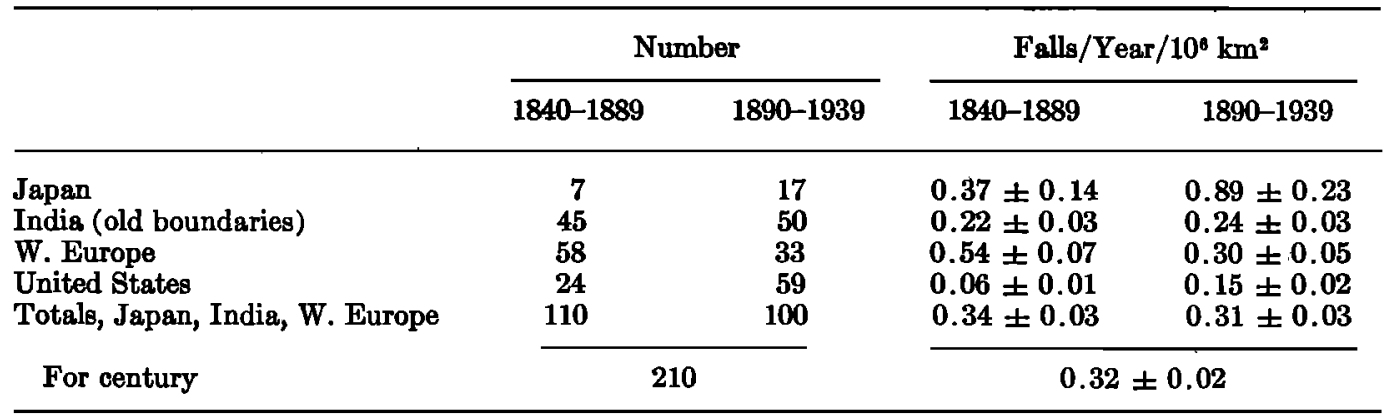

Area of earth $=510 \times 10^{6} \mathrm{~km}^{2}$.

Total 'falls' per year $=160$.

in the United States, although increasing, is very low, as might be expected on the basis of the relatively low population density.

It should be pointed out, however, that between the years 1923 and 1948, Nininger [1952] carried out an intensive program of search for witnessed falls in a limited area of $10^{\circ} \mathrm{km}^{2}$ in the central region of the United States. Over the 25-year period 13 witnessed falls were recovered, corresponding to 0.5 fall/year $/ 10^{\circ} \mathrm{km}^{2}$.

The figures indicate that with respect to the variable of population density, and ignoring for the moment other factors, the recovery of fallen meteorites in highly populated areas may be fairly efficient. The grand average for the rate of the fall in these areas is $0.32 \pm 0.02$ meteorite/year $/ 10^{\circ} \mathrm{km}^{2}$. This actually represents a lower limit for the rate of fall, for even in these areas a number of meteorites must fall that are not observed or recovered.

Taking the figure of 0.32 fall/year $/ 10^{\circ} \mathrm{km}^{2}$ for the density of observed falls, we arrive at the lower limit of 160 meteorite falls over the earth

TABLE 2. Classified Meteorite Falls Recorded (Prior's Catalogue, 1953)

\begin{tabular}{|c|c|c|}
\hline Class & Number & Per Cent \\
\hline Irons & 42 & 6.6 \\
\hline Stony irons & 12 & 1.9 \\
\hline Chondrites & 523 & 82.8 \\
\hline Achondrites & 56 & 8.7 \\
\hline Total & 633 & 100.0 \\
\hline
\end{tabular}

each year. The word 'fall' is used here to denote a meteorite that passes through the atmosphere and, after landing, is large enough to be found and picked up.

The distribution of observed falls among the major categories of meteorites is shown in Table 2.

It has long been known that there is a marked seasonal and diurnal variation in the rate of observed meteorite falls. The monthly variation in observed falls has a broad maximum ranging from April to July and a minimum ranging from October to March. There are two possible explanations for this variation: $(a)$ most of the observations originate in the northern hemisphere, and the summer months are better than the winter months for observing and recovering; (b) the earth may pass through a region which is dense with meteorites during the northern summer months.

Unfortunately the falls in the southern hemisphere are so few that statistics are poor. There does not appear to be a maximum coinciding with that observed in the northern hemisphere. Indeed, the numbers appear to support the existence of a truly seasonal effect, indicating that the number of daylight hours is important in the observation and recovery of falls. If we assume that the actual fall of meteorites upon the earth is not dependent upon the month, and that observations for May are typical of the entire year, the fall density computed above should be increased by 50 per cent.

The curve for the hour of fall has a pronounced peak between 15 and $16 \mathrm{~h}$ and drops to a minimum between 3 and $4 \mathrm{~h}$. In part, the 


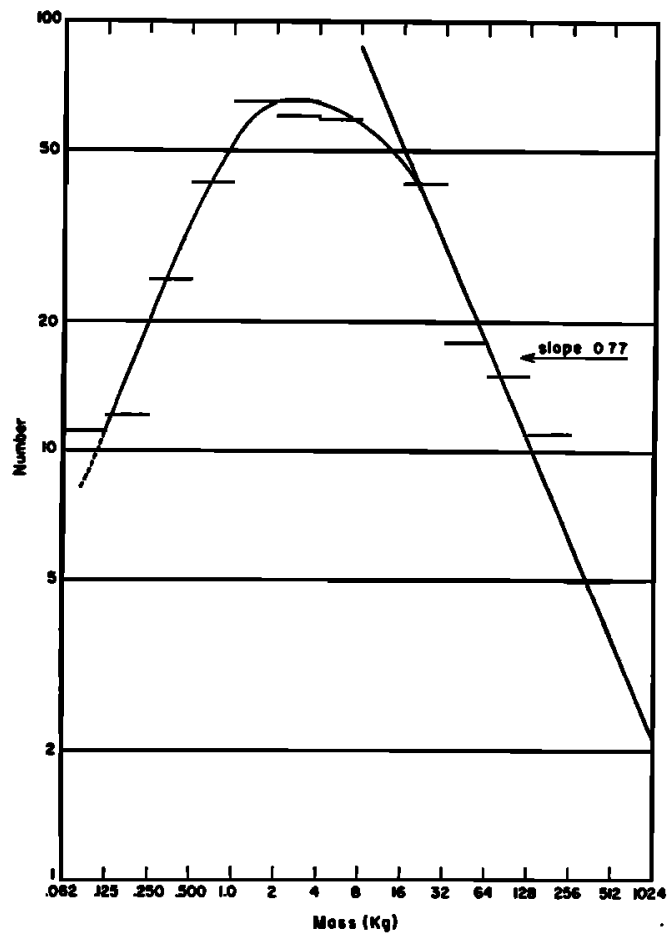

Fig. 1. Stone meteorite falls, sample 416.

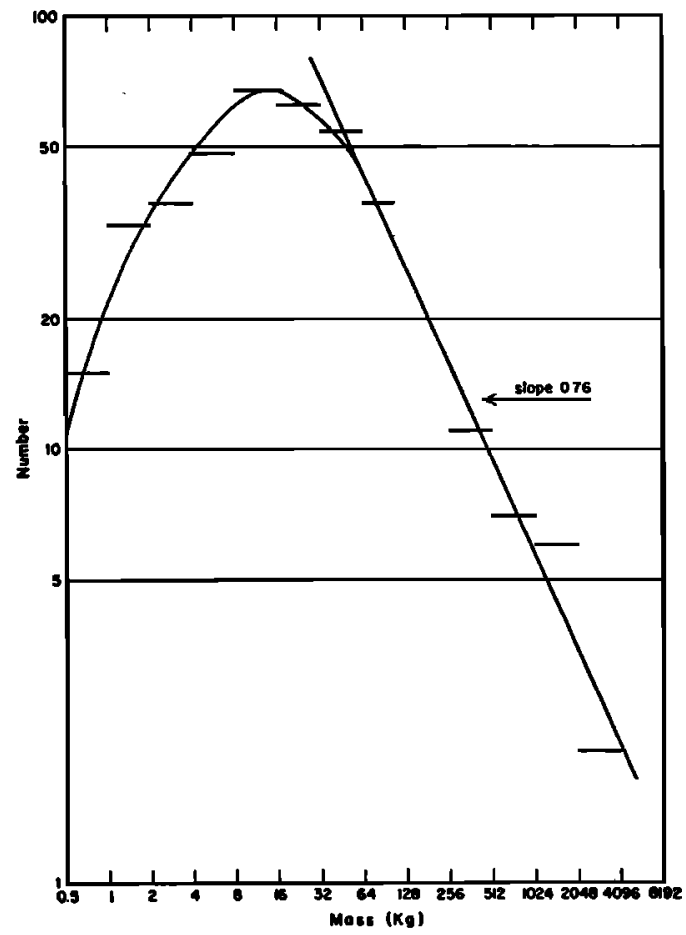

Fig. 2. Iron meteorite falls and finds, sample 399.

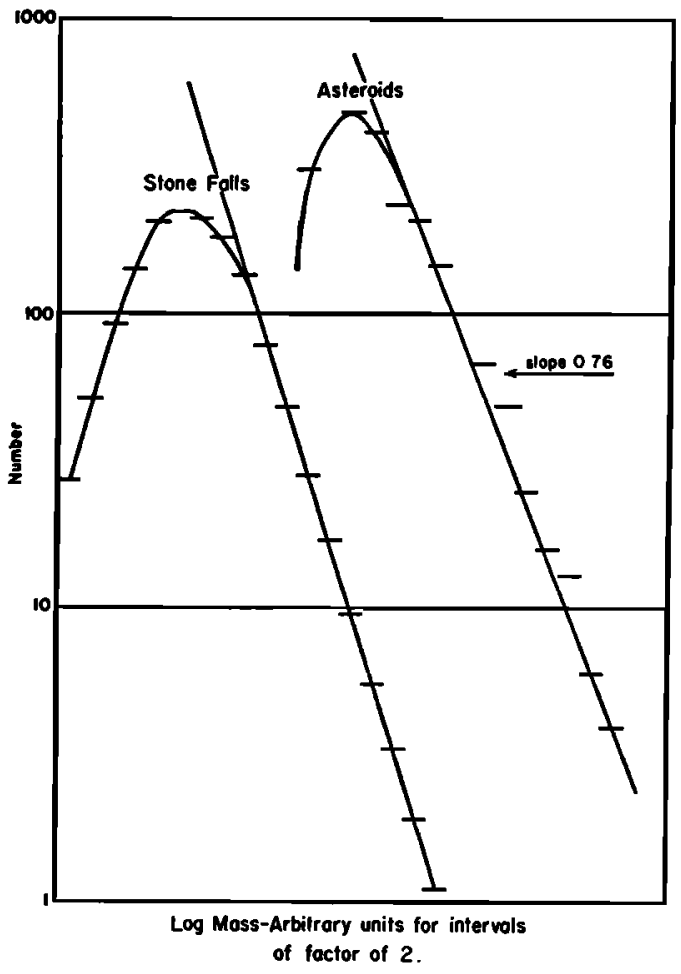

Fig. 3. Comparison of asteroids and meteorites.

time-of-fall curve can be explained on the basis of the greater probability of observing and recovering meteorites during the daylight hours, but other factors appear to be involved. If we assume that the actual fall of meteorites upon the earth is not dependent upon the time of day and that observations between 15 and $17 \mathrm{~h}$ are typical, the fall density computed above should be increased by an additional factor of 2 . Thus, the combination of the diurnal and seasonal effects could increase the calculated fall density from 0.32 to about 1.0 meteorite/year $/ 10^{\circ} \mathrm{km}^{2}$. Accordingly, the total number of world-wide 'falls' would be increased from 160 meteorites/ year to $\mathbf{4 8 0}$ meteorites/year.

Frequency distribution of meteorites with respect to mass. The frequency of stony meteorite falls as a function of mass is shown in Figure 1. It will be noted that as the mass increases the frequency passes through a maximum in the neighborhood of $1-4 \mathrm{~kg}$, after which the frequency falls off rapidly with increasing mass according to the relation

$$
(d \log M) /(d \log f)=0.77
$$




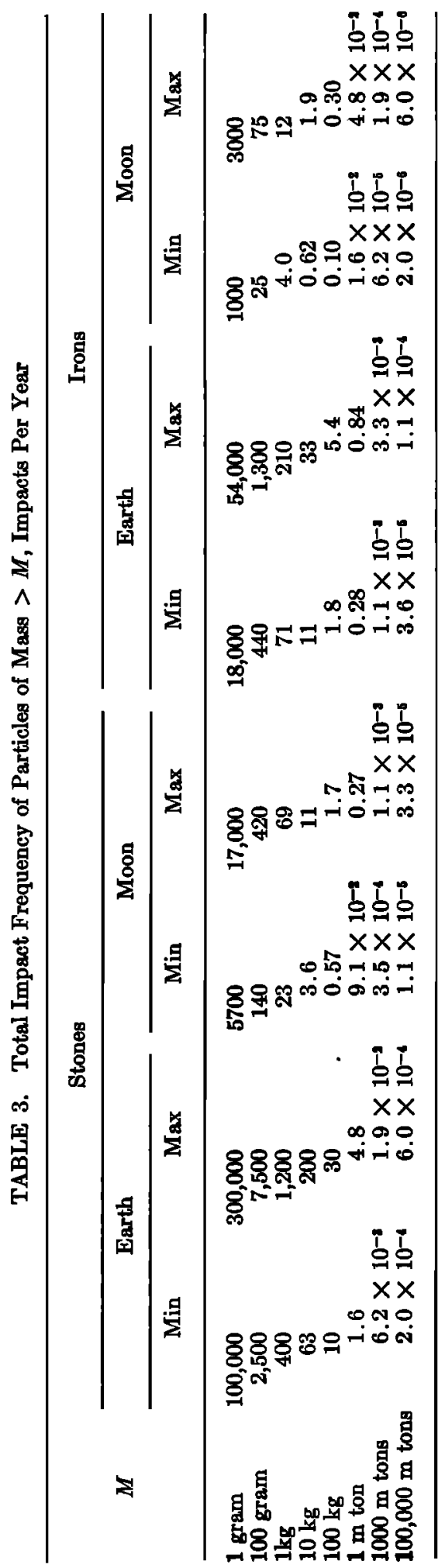


The frequency curve for iron meteorites, shown in Figure 2, is very nearly the same as that for stones. The curve passes through a maximum in the neighborhood of $8-32 \mathrm{~kg}$, then falls off steadily with a slope that appears to be identical with that of stone meteorites.

The frequency curve for stony meteorites deviates from this simple relationship at masses lower than about $16 \mathrm{~kg}$. That for iron meteorites deviates at masses lower than about $32 \mathrm{~kg}$. It is tempting to believe that the simple relationship holds over a much larger mass range and that deviations at low masses result in part from attrition in the atmosphere and in part from the lessened probability of seeing and recovering small bodies once they reach the earth. This suspicion is fortified by the striking resemblance of the frequency distribution curve as a function of magnitude for asteroids [Kuiper and others, 1958] to the curves for meteorites. If we assume a constant albedo and specific gravity for asteroids, a decrease of 0.5 in absolute magnitude corresponds to an increase by a factor of 2 in mass. The comparison of the observed asteroid curve with the smoothed curve for stony meteorites is shown in Figure 3.
Total meteorite impact upon the earth and moon as a function of mass. Assuming the simple relationship described above, the total frequency of meteorite impact upon the earth, calculated as a function of mass both for iron meteorites and for stones, is shown in Table 3. Two sets of values are given: the first set is uncorrected for seasonal and diurnal variations in recovery efficiency, and the second is corrected for these variations. The results, in turn, have been translated into collision frequencies with the moon. It is assumed that, because of the higher gravitational field of the earth, the frequency of impact upon the earth is increased by about 30 per cent above that calculated from geometry.

In space, the density of meteoritic bodies weighing 1 gram and more is calculated to be $10^{-19}$ particle $/ \mathrm{km}^{\mathrm{s}}$.

\section{REFERENCES}

Kuiper, G. P., and others, Astrophys. J. (Suppl. Ser.), s, 289, 1958.

Nininger, H. H., Out of the Sky, University of Denver Press, pp. 66-67, 1952.

Öpik, E. J., Irish Astron. J., 5, 34-36, 1957.

(Manuscript received March 31, 1960.) 